\title{
Control Strategy for Buck DC/DC Converter Based on Two-dimensional Hybrid Cloud Model
}

\author{
Qing-Yu Wang*, Ren-Xi Gong ${ }^{\dagger}$, Li-Wen Qin* and Zhao-He Feng*
}

\begin{abstract}
In order to adapt the fast dynamic performances of Buck DC/DC converter, and reduce the influence on converter performance owing to uncertain factors such as the disturbances of parameters and load, a control strategy based on two-dimensional hybrid cloud model is proposed. Firstly, two cloud models corresponding to the specific control inputs are determined by maximum determination approach, respectively, and then a control rule decided by the two cloud models is selected by a rule selector, finally, according to the reasoning structure of the rule, the control increment is calculated out by a two-dimensional hybrid cloud decision module. Both the simulation and experiment results show that the strategy can dramatically improve the dynamic performances of the converter, and enhance the adaptive ability to resist the random disturbances, and its control effect is superior to that of the current-mode control.
\end{abstract}

Keywords: Buck DC/DC converter, Uncertainty, Two-dimensional hybrid cloud model

\section{Introduction}

A DC/DC converter is a kind of power electronic converter and has been extensively used in many fields. In recent years, there have been manifested a great deal of control strategies associated with such a converter. According to whether strategy designs rely on converter models or not, they can be broadly divided into two categories as follows:

\subsection{Control strategies based on converter models}

In [1], the notion of the $N$-step model is introduced to capture the hybrid nature of DC/DC converters, and an optimal control problem is formulated and solved offline. The resulting state-feedback control law is of piecewise affine form, which can be easily stored and implemented in a lookup table and used for the practical implementation of the control strategy. In [2], a discrete controller for DC/DC converter is designed based on average model. Such a digital controller combined with the load estimator provides a complete compensation, which further improves the dynamic performance of the control system. In [3] and [4], the passivity-based control strategy is applied to the control of $\mathrm{DC} / \mathrm{DC}$ converter, which not only ensures the fast response of the system in different modes but also stabilizes output voltage in a wide range of operation. In addition, the differential geometry theory is used to the

\footnotetext{
$\dagger$ Corresponding Author: School of Electrical Engineering, Guangxi University, Nanning 530004, China. (rxgong@gxu.edu.cn)

* School of Electrical Engineering, Guangxi University, Nanning 530004, China. (wangqy@mail.gxu.cn, qinliwen901011@sina.com, f zhaohe@163.com)

Received: December 4, 2015; Accepted: May 25, 2016
}

modeling and control for DC/DC converter in [5] and [6]. This control strategy is based on the exact feedback linearization model of DC/DC converter, improving the robust and dynamic performances of the control system. Generally speaking, the above control strategies are all based on converter models which can be established through a variety of ways. Yet, the parameters of converter are sensitive to the disturbance of random factors, leading to the uncertainty and variability of the established model, which usually decreases the control performance and makes some control strategies too complicated to apply.

\subsection{Control strategies without requirement of a model}

When the construction and some parameters of a controlled object are uncertain, or its exact model cannot be established, some control strategies can also be designed by experience or field debugging, traditional PI control is one of them. Moreover, voltage-current two closed loops control [7], peak-current control [8,9], and average-current control [10], etc., also belong to the category. Although these strategies are simple and practical, they suffer from the effect of system noise and parameter disturbance, and hard to obtain the optimal dynamic performance (By the optimal dynamic performance here is meant both the relatively small adjusting time and overshoot), thus limiting their applications in some fields where the high performance is needed. In addition, for some intelligent control strategies such as neural network control [11] and fuzzy control [12], a model is also not required. Neural network control has the capability of supporting the linear or nonlinear mapping, as well as learning rules from the raw data, but the requirement of its real-time is still hard to be satisfied for the high switching frequency DC/DC 
converter. And a fuzzy control uses language description and rules to express the intuition and experience of designers directly so that a rapid and effective control can be realized even though the model of controlled object is unknown, but the effect of randomness is ignored. Some random factors will lead to the disturbance of the DC/DC converters during steady-state operation, thus degrading the fuzzy control performance.

Cloud is a model described by linguistic values for representation of the uncertain relationships between a specific qualitative concept and its quantitative expression, which integrates the fuzziness and randomness (two important factors of uncertainty) in a unified way [13]-[18]. For the controller design of a DC/DC converter, the mapping relationship between the input and output of the controller has uncertainty in the process of the converter operation. In such a case, how to regulate the output voltage to the expected value with relatively small adjusting time and overshoot is the main problem to be dealt with. In this paper, a control strategy for Buck DC/DC converter based on two-dimensional hybrid cloud model is proposed, which is suitable to tackle the above problem. Firstly, the related concepts of the cloud model and the structure block diagram of the control strategy are illustrated briefly, subsequently, each parts of the block diagram is described in detail, finally, the effectiveness of the strategy are verified by simulation and experiment.

\section{Continuous-time Model of Buck converter}

A Buck DC/DC converter consists of voltage source $v_{\mathrm{s}}$, switch transistor $Q$, diode $D$, inductor $l$, capacitor $c$ and load $r_{\mathrm{o}}$, of which the switch transistor $Q$ is controlled by the manipulated variable $d(k)$ for each switch period $k ; D$ acts as a wheel diode; the inductor $l$ is used to store and deliver energy; the capacitor $c$ is used to filter the ramp voltage across the load. The equivalent topology is shown in Fig. 1, where $r_{1}$ and $r_{\mathrm{c}}$ are respectively representative of the internal resistor of $l$ and $c$.

By taking $x(t)=\left[i_{1}(t) v_{0}(t)\right]^{T}$ as the system state vector, where $i_{1}(t)$ is the inductor current and $v_{0}(t)$ the output voltage, the system is described by the following continuous time state-space equation:

$$
\dot{x}(t)=A x(t)+B v_{s}(t) s
$$

In the above equation, if the switch $Q$ is on, then $s=1$,

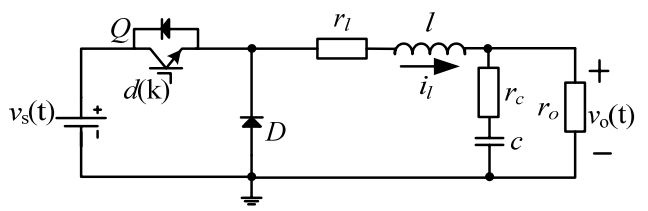

Fig. 1. Equivalent topology of the Buck DC/DC converter otherwise $s=0$, and matrices $A, B$ are given by

$$
A=\left[\begin{array}{cc}
-\frac{r_{l}}{l} & -\frac{1}{l} \\
\frac{-r_{o}\left(r_{c} c r_{l}-l\right)}{c l\left(r_{o}+r_{c}\right)} & \frac{-r_{c} r_{o} c-l}{c l\left(r_{o}+r_{c}\right)}
\end{array}\right] B=\left[\begin{array}{c}
\frac{1}{l} \\
\frac{r_{o} r_{c}}{\left(r_{o}+r_{c}\right) l}
\end{array}\right]
$$

\section{Concepts of Cloud Model}

Let $U$ be a universal set described by precise numbers, and $C$ be the qualitative concept related to $U$. If there is a number $x \in U$, which randomly realizes the concept $C$, and the membership degree of $x$ for $C$, i.e., $\mu(x) \in[0,1]$, is a random value with stabilization tendency

$$
\mu(x): U \rightarrow[0,1], \quad \forall x \in U, \quad x \rightarrow \mu(x)
$$

then the distribution of $x$ on $U$ is defined as a cloud [13]. Specially, provided that $\operatorname{NORM}_{1}\left(E_{1}, S_{1}\right)$ is a onedimensional normal distribution function, where $E_{1}$ is an expected value, $S_{1}$ is a standard deviation, then the cloud consists of cloud drops $\operatorname{drop}\left(x_{1 \mathrm{i}}, \mu_{\mathrm{i}}\right)(i=1,2 \ldots)$ which satisfy equation (4)

$$
\begin{gathered}
x_{1 i}=\operatorname{NORM}_{1}\left(E x_{x 1}, E n_{x 1}\right) \\
P_{1 i}=\operatorname{NORM}_{1}\left(E n_{x 1}, H e_{x 1}\right) \\
\mu_{i}=e^{-\frac{1}{2}\left(\frac{\left(x_{1 i}-E x_{x 1}\right)^{2}}{p_{1 i}^{2}}\right)}
\end{gathered}
$$

is called one-dimensional normal cloud [13], and denoted as $\left(E x_{\mathrm{x} 1}, E n_{\mathrm{x} 1}, H e_{\mathrm{x} 1}\right)$. The properties of a cloud are reflected by the three digital characteristics: expected value $\left(E x_{\mathrm{x} 1}\right)$, entropy $\left(E n_{\mathrm{x} 1}\right)$ and hyper-entropy $\left(H e_{\mathrm{x} 1}\right)$.

It is not difficult to extend a one-dimensional normal cloud to the two-dimensional case. Provided that $\mathrm{NORM}_{2}$ $\left(E_{1}, E_{2}, S_{1}, S_{2}\right)$ is a two-dimensional normal distribution function, where $E_{1}, E_{2}$ are expected values, $S_{1}, S_{2}$ are standard deviations, and given three digital characteristics: expected value $\left(E x_{\mathrm{x} 1}, E x_{\mathrm{x} 2}\right)$, entropy $\left(E n_{\mathrm{x} 1}, E n_{\mathrm{x} 2}\right)$ and hyperentropy $\left(H e_{\mathrm{x} 1}, H e_{\mathrm{x} 2}\right)$, then the cloud consists of cloud drops $\operatorname{drop}\left(x_{1 \mathrm{i}}, x_{2 \mathrm{i}}, \mu_{\mathrm{i}}\right)(i=1,2 \ldots)$ which satisfy equation (5):

$$
\begin{gathered}
\left(x_{1 i}, x_{2 i}\right)=\operatorname{NORM}_{2}\left(E x_{x 1}, E x_{x 2}, E n_{x 1}, E n_{x 2}\right) \\
\left(P_{1 i}, P_{2 i}\right)=\operatorname{NORM}_{2}\left(E n_{x 1}, E n_{x 2}, H e_{x 1}, H e_{x 2}\right) \\
\mu_{i}=e^{-\frac{1}{2}\left[\frac{\left(x_{1 i}-E x_{x 1}\right)^{2}}{p_{1 i}^{2}}+\frac{\left(x_{2 i}-E x_{x 2}\right)^{2}}{p_{2 i}^{2}}\right]}
\end{gathered}
$$

is called two-dimensional normal cloud [13], and it can be treated as the combination of two relatively independent one-dimensional normal cloud $\left(E x_{\mathrm{x} 1}, E n_{\mathrm{x} 1}, H e_{\mathrm{x} 1}\right)$ and $\left(E x_{\mathrm{x} 2}\right.$ $\left.E n_{\mathrm{x} 2}, H e_{\mathrm{x} 2}\right)$. 


\section{Proposed Control Strategy}

\subsection{Control structure based on Two-dimensional hybrid cloud model}

In Fig. 2 is shown the structure block diagram of implementing the proposed control strategy for a Buck DC/DC converter based on two-dimensional hybrid cloud model, where the output-voltage error $e(k)$ and the error increment $e c(k)$ are two inputs of the controller for each switch period $k$, of which $e(k)=U_{\text {ref }}-v_{0}(k), e c(k)=e(k)-$ $e(k-1)$, and $U_{\text {ref }}$ is the expected value of $v_{0}(k)$. The controller mainly consists of four parts: two maximum determination modules, a rule selector which contains a rule base, and a two-dimensional hybrid cloud decision module.

The implementation of the control strategy can be described as follows: firstly, the two one-dimensional cloud models $E_{\mathrm{i}}$ and $E C_{\mathrm{j}}$ (whose numerical representations are shown in Table 1) corresponding to $e(k)$ and $e c(k)$ are determined by the maximum determination modules, respectively, and then a control rule decided by the two clouds is selected by the rule selector from the rule base, and according to the reasoning structure of the rule and the digital characteristics of related cloud models, the control increment $\Delta d(k)$ can be calculated out by a twodimensional hybrid cloud decision module. Finally, the

Table 1. Numerical characteristics of one-dimensional hybrid cloud model sets

\begin{tabular}{|c|c|c|}
\hline $\begin{array}{l}\text { Qualitative } \\
\text { concepts }\end{array}$ & $\begin{array}{c}E=\left[E_{1}, E_{2}, \ldots E_{7}\right] \\
\text { cloud model set }\end{array}$ & $\begin{array}{c}E C=\left[E C_{1}, E C_{2}, \ldots E C_{7}\right] \\
\text { cloud model set }\end{array}$ \\
\hline NL & $\begin{aligned} E_{1}=1, & \text { if } \mathrm{x} \in[-4.5,-40], \\
& \text { otherwise } \\
E_{1}= & (-4.5,0.8,0.04)\end{aligned}$ & $\begin{aligned} E C_{1}=1, \text { if } \mathrm{x} \in[-0.6,-0.9], & \\
& \text { Otherwise } \\
E C_{1}= & (-0.6,0.08,0.004)\end{aligned}$ \\
\hline NM & $E_{2}=(-1.5,0.2,0.02)$ & $E C_{2}=(-0.3,0.03,0.004)$ \\
\hline NS & $E_{3}=(-0.5,0.1,0.02)$ & $E C_{3}=(-0.15,0.03,0.004)$ \\
\hline $\mathrm{ZE}$ & $E_{4}=(0,0.07,0.015)$ & $E C_{4}=(0,0.03,0.004)$ \\
\hline PS & $E_{5}=(0.5,0.1,0.02)$ & $E C_{5}=(0.15,0.03,0.004)$ \\
\hline PM & $E_{6}=(1.5,0.2,0.02)$ & $E C_{6}=(0.3,0.03,0.004)$ \\
\hline PL & $\begin{array}{c}E_{7}=1, \text { if } \mathrm{x} \in[4.5,40], \\
\text { otherwise } \\
E_{7}=(4.5,0.8,0.04)\end{array}$ & $\begin{array}{c}E C_{7}=1, \text { if } \mathrm{x} \in[0.6,0.9], \\
\text { otherwise } \\
E C_{7}=(0.6,0.08,0.004)\end{array}$ \\
\hline
\end{tabular}

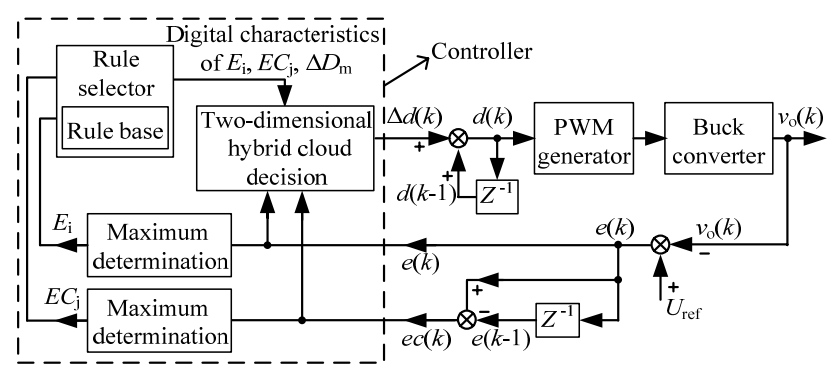

Fig. 2. Structure block diagram of implementing the proposed control strategy for a Buck DC/DC converter based on two-dimensional hybrid cloud model manipulated variable $d(k)$ is obtained by $d(k)=\Delta d(k)+d(k$ $1)$, and it is converted to the PWM control signal of switch transistor $Q$ by the PWM generator.

\subsection{Determination of cloud model}

First of all, two one-dimensional cloud model sets are respectively defined in the universal sets of $e(k)$ and $e c(k)$, denoted as $E=\left[E_{1}, E_{2}, \ldots E_{7}\right]$ and $E C=\left[E C_{1}, E C_{2}, \ldots E C_{7}\right]$. In $E$ and $E C$, the cloud models $E_{1}, E_{7}, E C_{1}$, and $E C_{7}$ are half trapezium cloud models [17], [18], while the others are normal cloud models, therefore, they are actually two onedimensional hybrid cloud model sets. The qualitative concept sets of $E$ and $E C$ are defined as (Negative Large (NL), Negative Medium (NM), Negative Small (NS), Zero (ZE), Positive Small (PS), Positive Medium (PM), Positive Large (PL) $\}$, and $\left(E x_{\text {ei }}, E n_{\text {ei }}, H e_{\text {ei }}\right),\left(E x_{\text {ecj }}, E n_{\text {ecj }}, H e_{\text {ecj }}\right)$ are utilized to denote the three digital characteristics of $E_{\mathrm{i}}, E C_{\mathrm{j}}$ $(i, j=1,2 \ldots 7)$, respectively. The numerical definitions are shown in Table 1.

Fig. 3 shows the distribution of $E$ and $E C$ yielding from Table 1, in which each cloud model consists of many cloud drops rather than certain function curve. The uncertainty of the cloud boundary reflects the fuzziness and randomness of a cloud model, but keeps the characteristics of a qualitative concept.

On defining the cloud model sets $E$ and $E C$, the following problem is to determine the two cloud models corresponding to the $e(k)$ and $e c(k)$ by maximum determination approach, respectively. The principle block diagram of the approach is shown in Fig. 4, where $\mathrm{CG}_{2}$ $\mathrm{CG}_{6}$ are one-dimensional normal cloud generators, and $\mathrm{HTCG}_{1}$ and $\mathrm{HTCG}_{7}$ are one-dimensional half trapezium

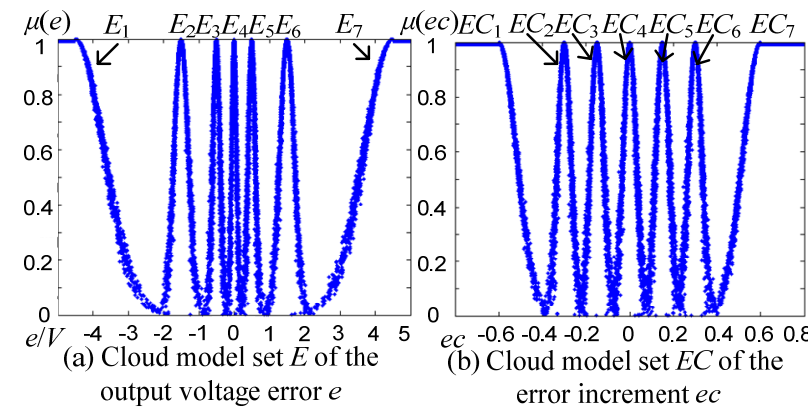

Fig. 3. One-dimensional hybrid cloud model sets of the voltage error $e$ and error increment $e c$

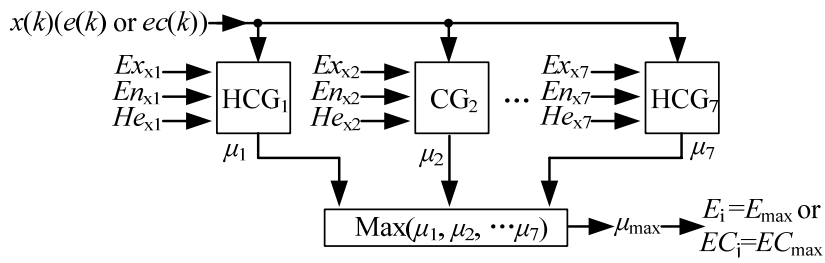

Fig. 4. Principle block diagram of maximum determination approach 
cloud generators. When each cloud generator is activated by the specific input $x(k)(e(k)$ or $e c(k))$, a corresponding membership degree $\mu_{\mathrm{i}}(\mathrm{i}=1,2, \ldots 7)$ is generated randomly. The maximal membership degree $\mu_{\max }$ can be then selected from the generated 7 membership degrees, and the cloud model corresponding to $x(k)$ is determined in terms of the subscript of $\mu_{\max }$.

\subsection{Rule selector}

In order to design the control rules, a one-dimensional normal cloud model set is defined in the universal set of control output $\Delta d(k)$, denoted as $\Delta D=\left[\Delta D_{1}, \Delta D_{2}, \ldots \Delta D_{7}\right]$, whose qualitative concept set is similar to that of $E$ and $E C$, and the numerical definition is in appendix.

According to the double-condition-single-rule reasoning structure: If $e(k)=E_{\mathrm{i}}$ and $e c(k)=E C_{\mathrm{j}}$, then $\Delta d(k)=\Delta D_{\mathrm{m}}(i$, $j, m=1,2, \ldots 7)$, and a control rule is determined. The intelligence of the control strategy is reflected by such rules. Following the expert experience, a rule base is established in Table 2, where the numbers 1 7 are used to briefly denote $E_{\mathrm{i}}, E C_{\mathrm{j}}, \Delta D_{\mathrm{m}}$, for example, $E_{\mathrm{i}}=3, E C_{\mathrm{j}}=5$, $\Delta D_{\mathrm{m}}=4$ corresponding to the control rule: If $e(k)=E_{3}(e(k)$ is NS) and ec $(k)=E C_{5}(e c(k)$ is PS $)$, then $\Delta d(k)=\Delta D_{4}(\Delta d(k)$ is $\mathrm{ZE}$ ).

After the control rule is selected by a rule selector, the digital characteristics of the related cloud models are subsequently provided to the two-dimensional hybrid cloud decision module.

\subsection{Two-dimensional hybrid cloud decision}

As can be seen in Fig. 5, the two-dimensional hybrid cloud decision module consists of a two-dimensional precondition hybrid cloud generator $\mathrm{HCG}_{\mathrm{Ei}, \mathrm{Ecj}}$ and a onedimensional postcondition cloud generator $\mathrm{CG}_{\Delta \mathrm{Dm}}$. It is used to realize the reasoning structure of a control rule.

The implementation of this module can be described as follows: firstly, by activating the $\mathrm{HCG}_{\mathrm{Ei}, \mathrm{Ecj}} n$ times with

Table 2. Rule base of two-dimensional cloud model

\begin{tabular}{c|c|c|c|c|c|c|c}
\hline & 1 & 2 & 3 & 4 & 5 & 6 & 7 \\
\hline $\mathrm{E}_{\mathrm{i}}$ & & & & & & & \\
\hline 1 & 1 & 1 & 1 & 1 & 2 & 3 & 4 \\
\hline 2 & 1 & 1 & 1 & 2 & 3 & 4 & 5 \\
\hline 3 & 1 & 1 & 2 & 3 & 4 & 5 & 6 \\
\hline 4 & 1 & 2 & 3 & 4 & 5 & 6 & 7 \\
\hline 5 & 2 & 3 & 4 & 5 & 6 & 7 & 7 \\
\hline 6 & 3 & 4 & 5 & 6 & 7 & 7 & 7 \\
\hline 7 & 4 & 5 & 6 & 7 & 7 & 7 & 7 \\
\hline
\end{tabular}

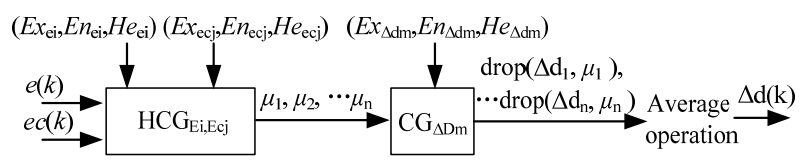

Fig. 5. Two-dimensional hybrid cloud decision module the same specific inputs $e(k)$ and $e c(k), n$ membership degrees $\mu_{1}, \mu_{2}, \ldots \mu_{\mathrm{n}}$ are generated randomly in terms of the two-dimensional hybrid cloud model (whose digital characteristics are decided together by that of the two one-dimensional cloud models, one of which is in $E$ and the other one is in $E C$ ). And then these membership degrees are used for the input of the $\mathrm{CG}_{\Delta \mathrm{Dm}}$ to generate $n$ cloud drops $\operatorname{drop}\left(\Delta d_{1}, \mu_{1}\right), \ldots d \operatorname{drop}\left(\Delta d_{\mathrm{n}}, \mu_{\mathrm{n}}\right)$ randomly. Finally, the control increment $\Delta d(k)$ is obtained by taking the mean value of $\Delta d_{1}, \ldots \Delta d_{\mathrm{n}}$. The two-dimensional hybrid cloud decision algorithm is illustrated below.

Algorithm 1 Two-dimensional hybrid cloud decision
Input: the numerical characteristics $\left(E x_{\mathrm{e}}, E n_{\mathrm{ei}}, H e_{\mathrm{ei}}, E x_{\mathrm{ecj}}\right.$,
$\left.E n_{\mathrm{ec}}, H e_{\mathrm{ecj}}\right)$ of the two-dimensional hybrid cloud model,
the numerical characteristics $\left(E x_{\Delta \mathrm{dm}}, E n_{\Delta \mathrm{dm}}, H e_{\Delta \mathrm{dm}}\right)$ of the
normal cloud model, two specific values $e(k)$ and $e c(k)$,
and the number $n$ of cloud drops.

Output: control increment $\Delta d(k)$.

Step 1: If $i=1$ and $e(k)<E x_{\mathrm{e} 1}$, then $e(k)=E x_{\mathrm{e} 1}$; If $i=7$ and $e(k)>E x_{\mathrm{e} 7}$, then $e(k)=E x_{\mathrm{e} 7} ;$ If $j=1$ and $e c(k)<E x_{\mathrm{ec} 1}$, then $e c(k)=E x_{\mathrm{ec} 1}$; If $j=7$ and $e c(k)>E x_{\mathrm{ec} 7}$, then $e c(k)=E x_{\mathrm{ec} 7} ; / *$ When $e(k), e c(k)$ correspond to half trapezium cloud models: $E_{1}, E_{7}, E C_{1}, E C_{7} *$ /

Step 2: Generate two normal random numbers $P_{\text {ei }}, P_{\text {ecj }}$ with expectations $\left(E n_{\mathrm{ei}}, E n_{\mathrm{ecj}}\right)$ and standard deviations $\left(H e_{\mathrm{ei}}\right.$, $\left.H e_{\text {ecj }}\right),\left(P_{\text {ei }}, P_{\text {ecj }}\right)=\operatorname{NORM} 2\left(E n_{\text {ei }}, E n_{\text {ecj }}, H e_{\text {ei }}, H e_{\text {ecj }}\right)$.

Step 3: Calculate $\mu_{n}=e^{-\frac{1}{2}\left[\frac{\left(e(k)-E x_{e i}\right)^{2}}{p_{e i}^{2}}+\frac{\left(e c(k)-E x_{e c j}\right)^{2}}{p_{e c j}^{2}}\right]}$.

Step 4: Repeat steps 2 3 until $n$ cloud drops are generated.

Step 5: Generate a normal random number $P_{\Delta \mathrm{dm}}$ with expectation $E n_{\Delta \mathrm{dm}}$ and standard deviation $H e_{\Delta \mathrm{dm}}, P_{\Delta \mathrm{dm}}=$ $\operatorname{NORM1}\left(E n_{\Delta \mathrm{dm}}, H e_{\Delta \mathrm{dm}}\right)$.

Step 6: If $e(k) \leq E x_{\mathrm{ei}}, e c(k) \leq E x_{\mathrm{ecj}}$, then $\Delta d_{\mathrm{n}}=E x_{\Delta \mathrm{dm}}-P_{\Delta \mathrm{dm}}$ $\times\left(-2 \ln \left(\mu_{\mathrm{n}}\right)\right)^{0.5}$;

elseif $e(k)>E x_{\mathrm{ei}}, e c(k)>E x_{\mathrm{ecj}}$, then $\Delta d_{\mathrm{n}}=E x_{\Delta \mathrm{dm}}+P_{\Delta \mathrm{dm}} \times$ $\left(-2 \ln \left(\mu_{\mathrm{n}}\right)\right)^{0.5}$;

elseif $e(k) \leq E x_{\mathrm{ei}}, e c(k)>E x_{\mathrm{ecj}}$, then $\mu^{\prime}=e^{\frac{-\left(e(k)-E x_{e i}\right)^{2}}{2 p_{e i}^{2}}}$,

$\Delta d_{\mathrm{n}}^{\prime}=E x_{\Delta \mathrm{dm}}-P_{\Delta \mathrm{dm}} \times\left(-2 \ln \left(\mu^{\prime}\right)\right)^{0.5}, \quad \mu^{\prime \prime}=e^{\frac{-\left(e c(k)-E x_{e c}\right)^{2}}{2 p_{e c j}^{2}}}$,

$\Delta d^{\prime \prime}{ }_{\mathrm{n}}=E x_{\Delta \mathrm{dm}}+P_{\Delta \mathrm{dm}} \times\left(-2 \ln \left(\mu^{\prime \prime}\right)\right)^{0.5}, \Delta d_{\mathrm{n}}=\left(\Delta d_{\mathrm{n}}^{\prime} \mu^{\prime}+\Delta d^{\prime \prime}{ }_{\mathrm{n}} \mu^{\prime \prime}\right) /$

$\left(\mu^{\prime}+\mu^{\prime \prime}\right)$;

elseif $e(k)>E x_{\mathrm{ei}}, e c(k) \leq E x_{\mathrm{ecj}}$, then $\mu^{\prime}=e^{\frac{-\left(e(k)-E x_{e i}\right)^{2}}{2 p_{e i}^{2}}}$,

$\Delta d_{\mathrm{n}}^{\prime}=E x_{\Delta \mathrm{dm}}+P_{\Delta \mathrm{dm}} \times\left(-2 \ln \left(\mu^{\prime}\right)\right)^{0.5}, \quad \mu^{\prime \prime}=e^{\frac{-\left(e c(k)-E x_{e c j}\right)^{2}}{2 p_{e c j}^{2}}}$,

$\Delta d^{\prime \prime}{ }_{\mathrm{n}}=E x_{\Delta \mathrm{dm}}-P_{\Delta \mathrm{dm}} \times\left(-2 \ln \left(\mu^{\prime \prime}\right)\right)^{0.5}, \Delta d_{\mathrm{n}}=\left(\Delta d_{\mathrm{n}}^{\prime} \mu^{\prime}+\Delta d^{\prime \prime}{ }_{\mathrm{n}} \mu^{\prime \prime}\right) /$

$\left(\mu^{\prime}+\mu^{\prime \prime}\right)$.

Step 7: Repeat steps 5 6 until $n$ cloud drops are generated. Step 8: Calculate the mean value of $\Delta d_{1}, \ldots \Delta d_{\mathrm{n}}, \Delta d(k)=$ mean $\left(\Delta d_{1}, \ldots \Delta d_{\mathrm{n}}\right)$. 


\section{Simulation and Experiment}

\subsection{Converter and control parameters}

In order to verify the control performance of the proposed control strategy, a simulation platform and an experiment platform of the Buck DC/DC converter are constructed, and both the simulation and experiment results are compared with that of the current-mode control. The converter parameters used are shown in Table 3.

In Fig. 6 is shown the structure block diagram of a common current-mode controlled Buck DC/DC converter. For PWM duty cycles greater than $50 \%$, the current-mode control requires a slope compensation signal $v_{\text {ramp }}$ to avoid sub-harmonic oscillation [19]. According to the parameters in Table 3 and related formulas in [19], the minimum compensation slope $m_{\mathrm{cmin}}=3333.3$ can be calculated out. The control parameters are chosen as $m_{\mathrm{c}}=3500$ for currentmode control and $k_{\mathrm{p}}=0.8, T_{\mathrm{i}}=0.002$ for PI control.

\subsection{Random circuit parameters disturbance}

In the process of the converter operation, the circuit parameters may change because of the disturbance and effect of uncertain factors such as noise and temperature. Here, the effect of random inductance parameters disturbance is mainly discussed, and the dynamic behavior of the converter during startup is investigated. In Fig. 8 is shown the simulation results under the effect of the disturbance signals shown in Fig. 7, and in Table 4 is summarized the evaluation parameters of control performance.

As can be seen in Table 4, the proposed control strategy not only improves the dynamic performances (both the

Table 3. Converter parameters

\begin{tabular}{cc||cc}
\hline parameters & values & parameters & values \\
\hline$l / \mathrm{mH}$ & 1.5 & $\mathrm{c} / \mathrm{uF}$ & 220 \\
$r_{\mathrm{l}} / \Omega$ & 1 & $r_{\mathrm{c}} / \Omega$ & 0.5 \\
$r_{\mathrm{o}} / \Omega$ & 20 & $v_{\mathrm{s}} / \mathrm{V}$ & 50 \\
$f_{\mathrm{s}} / \mathrm{kHz}$ & 20 & $U_{\mathrm{ref}} / \mathrm{V}$ & 30 \\
\hline
\end{tabular}

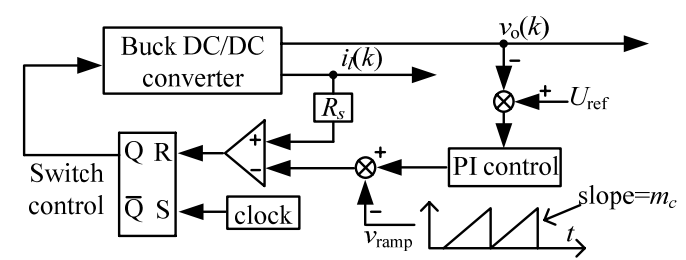

Fig. 6. Structure block diagram of current-mode controlled Buck DC/DC converter

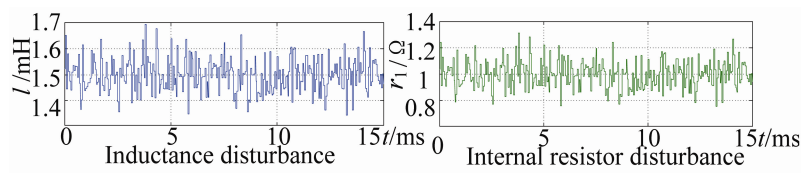

Fig. 7. Random inductance parameters disturbance signals
Table 4. Evaluation parameters of control performance

\begin{tabular}{c|c|c|c}
\hline Control strategy & $\begin{array}{c}v_{\mathrm{o}}, i_{1} \text { adjusting } \\
\text { time/ms }\end{array}$ & $\begin{array}{c}v_{\mathrm{o}} \\
\text { overshoot } / \%\end{array}$ & $\begin{array}{c}i_{1} \\
\text { max value } / \mathrm{A}\end{array}$ \\
\hline Cloud model control & 5.5 & 0 & 2.6 \\
\hline Current-mode control & 6 & 6.7 & 12.5 \\
\hline
\end{tabular}

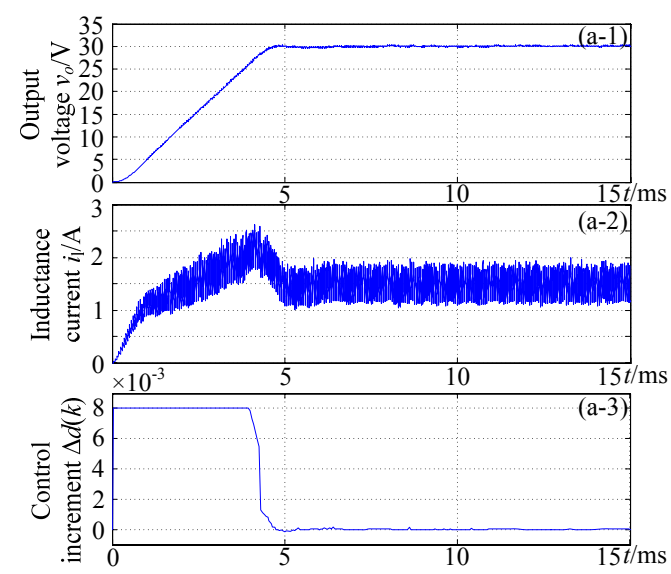

(a) Results obtained by the proposed control strategy

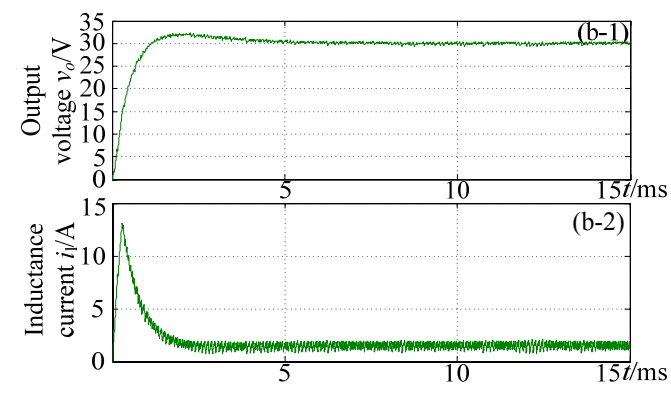

(b) Results obtained by the current-mode control

Fig. 8. Comparison of the results obtained by the proposed control strategy and current-mode control during startup

adjusting time and overshoot are relatively small), but also remarkably reduces the peak value of $i_{1}$ compared with the current-mode control. Fig. 8(a-3) shows that the whole adjusting process of $\Delta d(k)$ is smooth. Therefore, the control strategy is suitable for dealing with the random parameters disturbance. Moreover, the advantage of using two-dimensional hybrid cloud model is obvious, that is, the step size of $\Delta d(k)$ is maximum when $v_{\mathrm{o}}$ is far from $U_{\text {ref }}$, and only when $v_{\mathrm{o}}$ is close to $U_{\text {ref }}$, will the step size of $\Delta d(k)$ reduce gradually. Therefore, the adjusting time can be decreased and the error adjusting range can be extended.

As can be seen from the waveform details during startup in the interval of $11 \sim 15 \mathrm{~ms}$ in Fig. 9, the random parameters disturbance has a great influence on the currentmode control so as to cause large deviations in $v_{\mathrm{o}}$ and $i_{1}$. The maximum voltage deviation reaches $\pm 0.5 \mathrm{~V}$, while the fluctuation range of $i_{1}$ is also as great as $0.6 \sim 2 \mathrm{~A}$. By contrast, the proposed control strategy has strong antiinterference ability, and can make the maximum voltage 


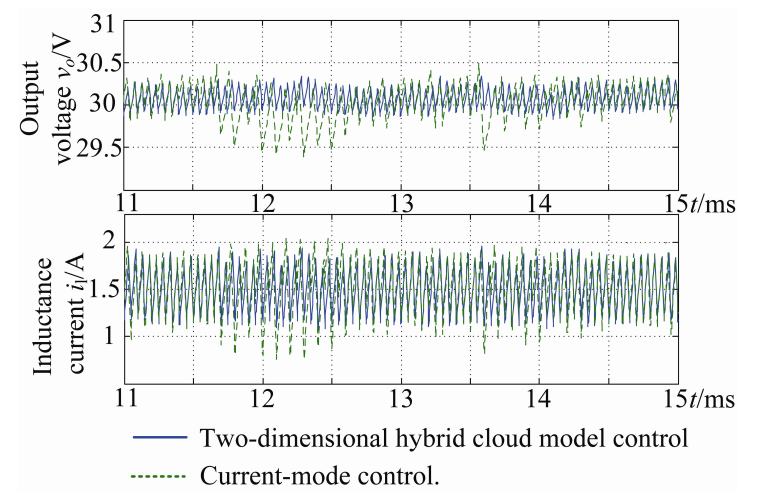

Fig. 9. Waveform details during startup

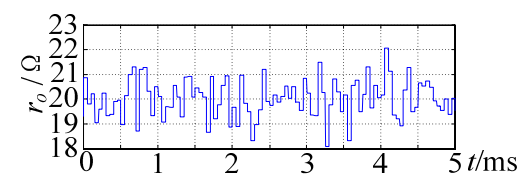

Fig. 10. Random load disturbance signal

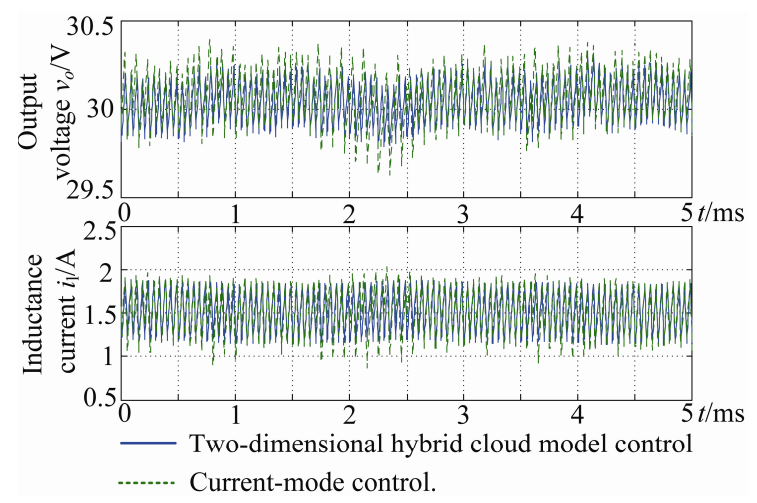

Fig. 11. Comparison of the results obtained by the two control strategies under random load disturbance

deviation less than $\pm 0.2 \mathrm{~V}$, and keeps $i_{1}$ within 1.1 1.9 A.

\subsection{Random load disturbance}

In order to compare the performance of the two control strategies under random load disturbance, a random signal shown in Fig. 10 is taken as the load disturbance.

The simulation results of $v_{\mathrm{o}}$ and $i_{1}$ under the random load disturbance are shown in Fig. 11. For current-mode control, it can be seen that the maximum voltage deviation reaches $\pm 0.3 \mathrm{~V}$, and $i_{1}$ has a big fluctuation. By contrast, the proposed control strategy not only keeps the voltage deviation within $-0.2 \sim 0.2 \mathrm{~V}$, but also makes $i_{1}$ without obvious fluctuation. This shows that the control strategy is also suitable for dealing with the random load disturbance.

\subsection{Experimental validation}

To further investigate the potential advantages of the

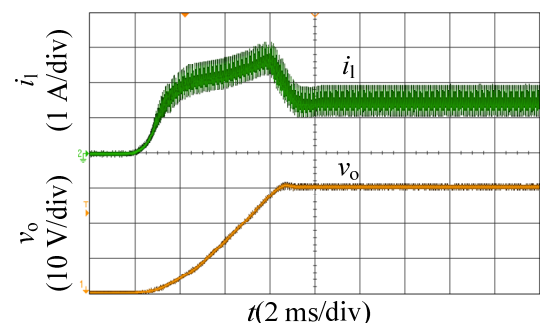

(a) Waveforms obtained by the proposed control strategy

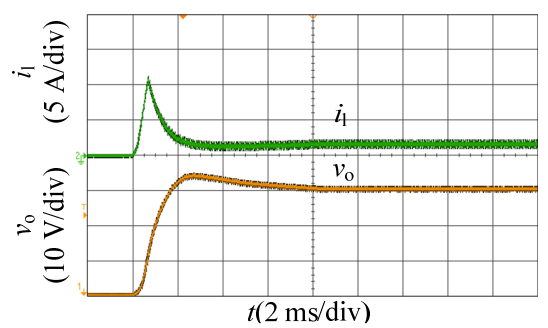

(b) Waveforms obtained by the current-mode control

Fig. 12. Comparison of the startup behavior

proposed control strategy, the experimental platform of the controller is constructed on an OP5600 RT-LAB real-time system, where OP5600 allows the development of the control software in a MATLAB/SIMULINK environment. Access to the input/output (I/O) of the real-time hardware is implemented through Real Time Workshop blocks. The Buck DC/DC converter is constructed by using an MOSFET (IRFP4468) as an active switch and a diode (10SQ80) as a passive switch. The startup behavior is obtained by a DC power analyzer N6705, and the load disturbance is realized by an electronic load N3306A. The control structure is shown in Fig. 2.

Fig. 12 shows the inductance current and the output voltage of the converter during startup. As can be seen, the proposed control strategy is more efficient than the currentmode control for improving the dynamic performances of the converter, that is, unnoticeable overshoot of $v_{\mathrm{o}}$, smaller peak of $i_{1}$, and shorter adjusting time of both $v_{\mathrm{o}}$ and $i_{1}$.

Furthermore, the waveform details during steady-state operation in Fig. 13 show that the control strategy can effectively restrain the random fluctuation of $v_{\mathrm{o}}$ and $i_{1}$. This proves the effectiveness of the proposed strategy for dealing with the random disturbance, and through observation and analysis, it can be seen that the random fluctuation in the experiment mainly results from the DC power supply.

In a last step, the control performance is investigated in the presence of step change in the load resistance. Starting from the empty-load, a step change to the full-load is applied, and the careful observation of the response is made. The condition observed is shown in Fig. 14. It can be seen that when a large load disturbance occurs, for the proposed control strategy, $v_{\mathrm{o}}$ can be quickly regulated to the expected value without steady-state error, while for the current-mode control, longer adjusting time is needed and a 


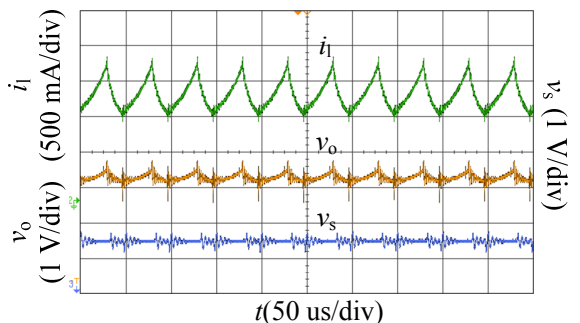

(a) Waveforms obtained by the proposed control strategy

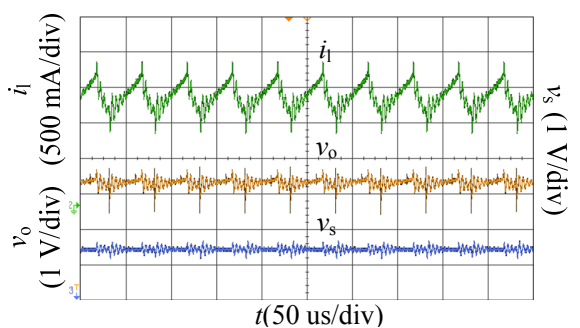

(b) Waveforms obtained by current-mode control

Fig. 13. Waveform details during steady-state operation

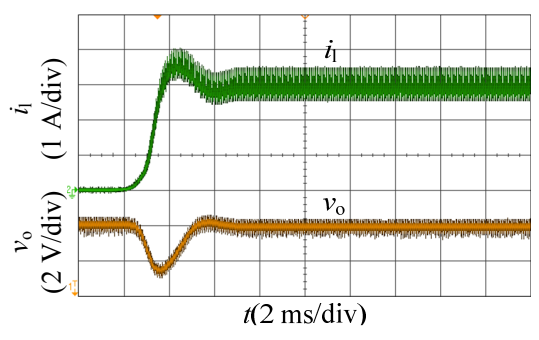

(a) Waveforms obtained by the proposed control strategy

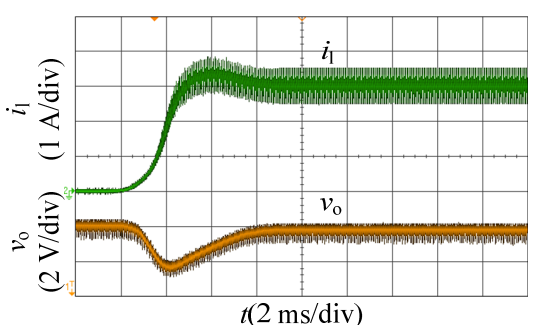

(b) Waveforms obtained by current-mode control

Fig. 14. Experiment waveforms with load disturbance

small steady-state error for $v_{\mathrm{o}}$ is appeared after the load disturbance.

\section{Conclusion}

For the control design of a converter, the mapping relationship between the input and output of the controller has uncertainty in the process of the converter operation, while cloud models are suitable for tackling this uncertainty relationship. Therefore, a control strategy for Buck DC/DC converter based on two-dimensional hybrid cloud model is proposed, which has the following properties: the control structure is simple, and easy to realize by digital control. In the process of the control design, the most important part is the algorithm design of the two-dimensional hybrid cloud decision module (Algorithm 1) which is used to realize the reasoning structure of a control rule. In the view of the computational complexity, the algorithm mainly consists of some judging syntax and index calculations, and the complex calculations are not involved so that the real-time control can be guaranteed, and the algorithm design can be easily implemented. By contrast, in the process of the current-mode control design, an appropriate slope compensation signal is needed to avoid sub-harmonic oscillation when the PWM duty cycle is greater than 50\%. Either the overcompensation signal or undercompensation signal may lead to the system instability. Therefore, there is no doubt that the calculation and generation of the slope compensation signal will make the control design and implementation more complicated. In addition, both the results obtained by simulation and experiment show that the current-mode control strategy is difficult to obtain the optimal dynamic performance, while the proposed control strategy can not only improve the dynamic performances of Buck DC/DC converter, but also has obvious advantages in dealing with the uncertain disturbance factors.

\section{Acknowledgements}

This study was supported by National Natural Science Foundation of China (No. 61561007) and Natural Science Foundation of Guangxi Province (No. 2014GXNSFBA 1182 77) and Innovation Project of Guangxi Graduate Education (No. YCBZ2015020).

\section{Appendix}

$\Delta D=\left[\Delta D_{1}, \Delta D_{2} \ldots \Delta D_{7}\right]$, where $\Delta D_{1}=(-0.008,0.002$, $0.0001) ; \Delta D_{2}=(-0.002,0.0002,0.00005) ; \Delta D_{3}=(-0.001$, $0.0002,0.00005) ; \Delta D_{4}=(0,0.0001,0.00002) ; \Delta D_{5}=(0.001$, $0.0002,0.00005) ; \Delta D_{6}=(0.002,0.0002,0.00005) ; \Delta D_{7}=$ $(0.008,0.002,0.0001)$.

\section{References}

[1] Tobias Geyer, Georgios Papafotiou and Manfred Morari, "Hybrid Model Predictive Control of the Step-Down DC/DC Converter," IEEE Trans. Control Systems Technology, vol. 16, no. 6, pp. 1112-1124, NOVE. 2008.

[2] R. Shenbagalakshmi, and T. Sree Renga Raja, "Implementation of Robust Prediction Observer Controller for DC/DC Converter" Journal of Electrical Engineering \& Technology, vol. 8, no. 6, pp. 1389-1399, Aug. 2013. 
[3] Mahdi Salimi, and Armin Lotfi Eghlim, "PassivityBased Control of the DC/DC Buck Converters in High-Power Applications," TENCON 2014-2014 IEEE Region 10 Conference, Bangkok, Thailand, Oct. 2014.

[4] Zeng Jian-wu, Zhang Zhe, and Qiao Wei, “An Interconnection-Damping-Assignment Passivity-Based Controller for a DC/DC Boost Converter with a Constant Power Load," IEEE Industry Applications Society Annual Meeting, Las Vegas, USA, Oct. 2012.

[5] Shuai Ding-xin and Xie Yun-xiang, "Optimal Control of Buck Converter by State Feedback Linearization," 7th World Congress on Intelligent Control and Automation Conference, Chongqing, China, June. 2008.

[6] Shuai Ding-xin, "State feedback exact linearization control of Buck-Boost converter," International Electronics and Application Conference and Exposition (PEAC), Shanghai, China, June. 2014.

[7] Yasuhiro Sugimoto, Toru Sai, Kei Watanabe, and Mikio Abe, "Feedback Loop Analysis and Optimized Compensation Slope of the Current-Mode Buck DC/DC Converter in DCM," IEEE Trans. Circuits and Systems, vol.62, no.1, pp. 311-319, Jun. 2015.

[8] N. Kondrath, and M.K. Kazimierczuk, "Control current and relative stability of peak current-mode controlled pulse-width modulated dc-dc converters without slope compensation," IET Power Electronics, vol. 3, no. 6, pp. 936-946, Mar. 2010.

[9] Kavitha, Anbukumar, and Uma, Govindarajan, "Resonant Parametric Perturbation Method to Control Chaos in Current Mode Controlled DC/DC BuckBoost Converter" Journal of Electrical Engineering \& Technology, vol. 5, pp. 171-178, Mar. 2010.

[10] Chia-Ling Wei, Chin-Hong Chen, Kuo-Chun Wu, and I-Ting Ko, "Design of an Average-Current-Mode Noninverting Buck-Boost DC/DC Converter With Reduced Switching and Conduction Losses," IEEE Trans. Power Electronics, vol. 27, no. 12, pp. 49344943, Dec. 2012.

[11] Hidenori Maruta, Masashi Motomura, and Fujio Kurokawa, "An Evaluation Study on Circuit Parameter Conditions of Neural Network Controlled DC/DC Converter," 12th International Conference on Machine Learning and Applications, pp. 249-254, Dec. 2013.

[12] Boumediene, and Allaoua, "Application of a Robust Fuzzy Sliding Mode Controller Synthesis on a BuckBoost DC-DC Converter Power Supply for an Electric Vehicle Propulsion System," Journal of Electrical Engineering \& Technology, vol. 6, no. 1, pp. 67-75, June. 2011.

[13] Li De-yi and Du Yi, Uncertainty of artificial intelligence: Chapman and Hall/CRC, 2007, p. 107150.

[14] Li De-yi, Shi Xue-mei and Ward P, "Soft inference mechanism based on cloud models," Proc of 1st Int Workshop on Logic Programming and Soft Computing, Bonn, Germany, June. 1996.

[15] Wang Guo-yin, Xu Chang-lin, and Li De-yi, "Generic normal cloud model," Information Sciences, vol.280, no.1, pp. 1-15, Oct. 2014.

[16] Li De-yi, Liu Chang-yu, and Gan Wen-yan, "A New Cognitive Model: Cloud Model," International Journal of Intelligent Systems, vol.24, pp. 357-375, 2009.

[17] Li De-yi, Cheng D W, and Shi Xue-mei, "Uncertainty reasoning based on cloud models in controllers," Journal of Computer Science and Mathematics with Application, vol.35, no.3, pp. 99-123, Apr. 1997.

[18] Jian Qiang Wang, Pei Wang, Jing Wang, Hong-Yu Zhang, and Xiao-Hong Chen, "Atanassov's IntervalValued Intuitionistic Linguistic Multicriteria Group Decision-Making Method Based on the Trapezium Cloud Mode," IEEE Trans. Fuzzy Systems, vol.23, no.3, pp. 542-554, JUNE. 2015.

[19] Guo Zhongjie, Wu Longsheng, and Liu Youbao, "Design and implementation of adaptive slope compensation in current mode DC/DC converter," Journal of Semiconductors, vol.31, no.12, pp. 1-7, Dec. 2010.

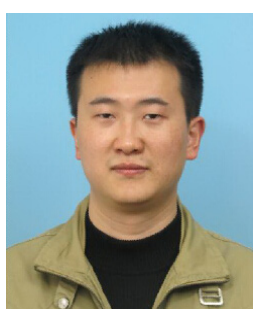

Qing-Yu Wang was born in Guizhou Province, China, in 1988. He received his B.S. degrees in Electronic Science and Technology from Guangxi University, Nanning, China in 2011. He is currently working on his Ph.D. in electric power system and automation in the same university. His research interests are nonlinear dynamics, the application of cloud model theory and hybrid system theory in power electronics.

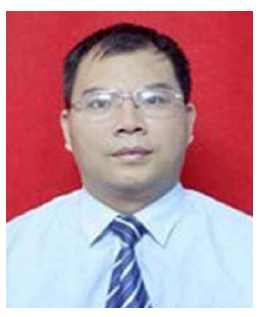

Ren-Xi Gong was born in Guilin of Guangxi Province in September 1962. From August 1990 to March 1993, he was a graduate student majoring in Semiconductor Devices and Microelectronics at Xidian University. $\mathrm{He}$ received his M.S. degree in March 1993. From 1994 to 1997, he worked as an assistant at Guangxi University, and from February 1998 to January 2002, he worked on his Ph.D degree in micro-electronics and solid-state electronics at Xidian University. He is currently a professor at Electrical Engineering School of Guangxi University. His present research interests are intelligent detection technology, power electronics and their applications. 


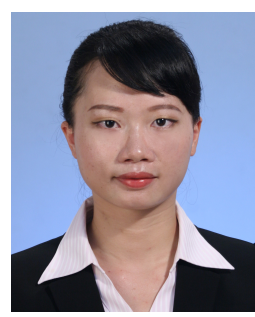

Li-Wen Qin was born in Guangxi Province, China, in 1990. She received her B.S. degrees in Automation of Electric Power System from Guangxi University, Nanning, China in 2013 and received her M.S. degree in 2016 in the same university. Her research interests are the application of cloud model theory in power system and the analysis of power system reliability.

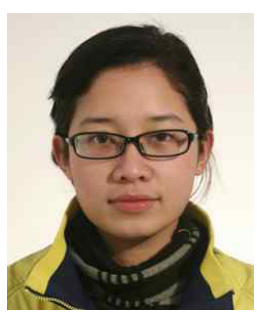

Zhao-He Feng was born in Guangxi Province, China, in 1986. She received the B.S. degrees in Automation from Beifang University of Nationalities, Yinchuan, China, in 2010. She is currently working on her Ph.D. in electric power system and automation in Guangxi University, Nanning, China. Her research interests are nonlinear dynamics, stability analysis of hybrid systems, and application of power electronics in renewable energies. 